\title{
Calculation of displacements in a half-space from a linear array based on analytical solutions of transient wave
}

\author{
Xuecong Zhang ${ }^{1}$, Cunfu $\mathrm{He}^{1}$, Yan $\mathrm{Lu}^{1, \mathrm{a}}$ and Bin $\mathrm{Wu}^{1}$ \\ ${ }^{1}$ College of Mechanical Engineering and Applied Electronics Technology, Beijing University of Technology, Beijing, \\ China
}

\begin{abstract}
In this paper, we propose a theoretical calculation method for the displacements of transient wave excited by a linear array coupled to an elastic solid with a longitudinal wave couplant. Firstly, the vertical and horizontal displacements excited by a vertical line source are solved based on exact and analytical solutions of transient elastic waves induced by a line source normally acting on an elastic half-space. Secondly, in this case, the displacements from a linear array can be determined by the superposition of the solutions of a number of discrete line forces mentioned above. All the theoretical results are compared to the finite element simulation results. The theoretical results are consistent with finite element simulation data. Therefore, the calculation method can save a lot of computing time. This paper provides a fast and accurate calculation method of transient wave displacements in a half-space generated by a linear array. The calculation method can be used to explore the sound field characteristics of a linear array transducer in the future.
\end{abstract}

\section{Introduction}

The ultrasonic array includes many active transducer elements which can be excited individually or simultaneously. Ultrasonic array has the flexibility to perform inspections along different directions with controllable beam patterns. These advantages accelerated the development of ultrasonic linear arrays for engineering and industry applications in the past several decades [1].

For the scanning over a larger area, a linear array transducer can be coupled to a sample with a longitudinal wave couplant, as shown in Fig. 1. Because the transient wave displacements in the detected object excited by the linear array can indicate characteristics of sound field, so it's very important to the transducer's design. As shown in Fig. 1, transducer elements are coupled to a sample with a longitudinal wave couplant. The inter-element spacing is $p$; element width is $l$; total element number is $N$; array size is $L(=N \cdot p)$. In the previous studies, many great contributions to ultrasonic transducer sound field calculation have been made[2-8]. Wooh et al. regarded each transducer element as many discrete line sources loading on the surface of a elastic half-space, to predict the ultrasound field radiated by the linear phased array [4-6]. It assumed that the sound wave from each line source could be modeled as a cylindrical wave generated from an infinitely long line source pulsating radially and harmonically in a unbounded acoustic medium.

\footnotetext{
${ }^{\text {a }}$ Corresponding author: lvyan@bjut.edu.cn
} 


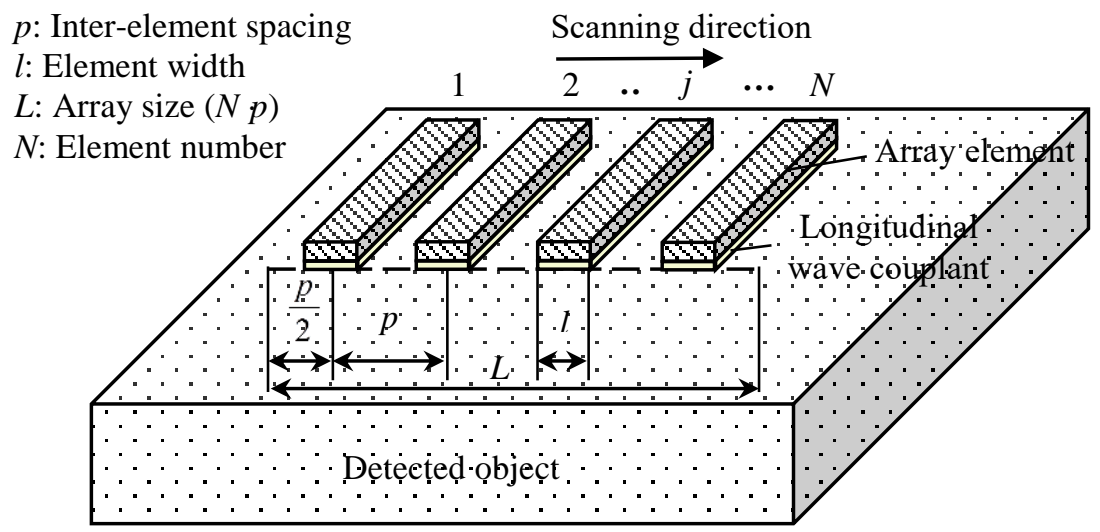

Figure 1. Geometry of a linear phased array transducer scanning over the surface of an object using longitudinal wave couplant.

In previous studies, theoretical calculation of ultrasound fields was largely simplified: First of all, for a vertical line force acting on a solid half-space, both longitudinal and shear waves are generated inside the solid and none of them can be characterized by a simple cylindrical wave with equal amplitude along all directions. Secondly, in real applications, transducer elements are not actuated by harmonic excitation but by pulsed waveforms with finite duration.

Consequently, the sound field based on analytical solutions of transient wave could provide precise characteristics of a linear array transducer. In this paper, we propose a theoretical calculation method for the displacements of transient wave excited by a linear array coupled to an elastic solid with a longitudinal wave couplant. Firstly, the vertical and horizontal displacements excited by a line source normally loading on an elastic half-space are solved by analytical solutions. Secondly, in this case, the displacements from a linear array can be determined by the superposition of the transient elastic wave displacements induced by a number of discrete line sources mentioned above. All the theoretical results are compared to the finite element (FE) simulation results and the consistent comparison results are obtained. This paper provides a fast and accurate calculation method for the transient displacements in a half-space generated by a linear array. The calculation method will be used to guide the design of a linear array transducer in the future.

\section{Transient wave displacements in an elastic solid half-space from a vertical line source loading on the surface}

For an ultrasonic linear array coupled to the surface of an elastic half-space with a longitudinal wave couplant, elastic waves are excited by the piezoelectric elements and transmitted to the elastic solid through normal stresses acting on the surface. Consequently, we should focus on the analytical solutions of elastic waves generated by a line vertical force loading on an elastic half-space, and derive the displacements of this transient elastic wave. As shown in Figure 2, a concentrated force is normally applied at a point of an elastic half-space defined in a Cartesian coordinate $(x, y)$, where $Q$ is a constant amplitude and $f(t)$ is an arbitrary function of time $t$. 


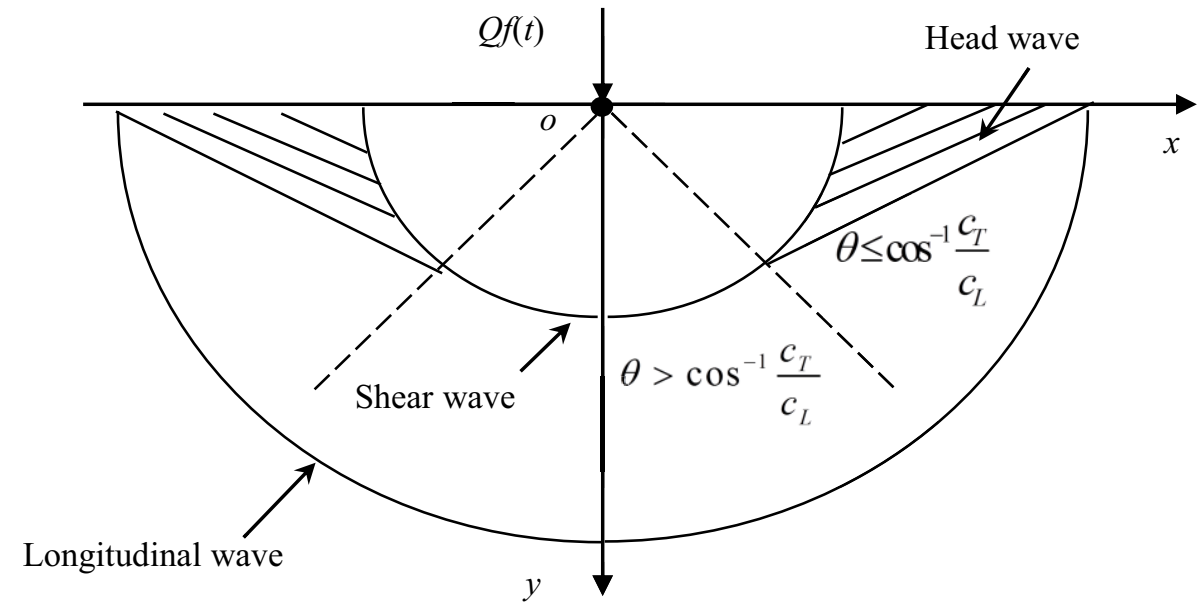

Figure 2. Wave fronts pattern from a vertical line force loading on an elastic half-space.

The problem has been studied thoroughly before[9]. The displacement components analytical solutions are shown as

$$
\begin{gathered}
u(x, y, t)=-\frac{Q}{\mu}\left[(u)_{L}-(u)_{T L}-(u)_{T}\right] \\
v(x, y, t)=\frac{Q}{\mu}\left[(v)_{L}+(v)_{T L}+(v)_{T}\right]
\end{gathered}
$$

where $u(x, y, t)$ is the horizontal displacement, $v(x, y, t)$ is the vertical displacement. $(u)_{L}$ and $(v)_{L}$ are longitudinal wave terms, $(u)_{T}$ and $(v)_{T}$ are shear wave terms, $(u)_{T L}$ and $(v)_{T L}$ are head wave terms, $\mu$ is the shear modulus of the elastic solid. The analytical solutions of $(u)_{L},(u)_{T L},(u)_{T},(v)_{L}$, $(v)_{T L}$ and $(v)_{T}$ could be obtained form Ref. [9]. The pattern of waveforms is shown in Figure 2. As shown in the figure, three modes exist: longitudinal wave, shear wave and head wave. Head wave only exists in the area where $\theta \leq \cos ^{-1} \frac{S_{L}}{S_{T}}$.

Figure 3 and 4 show the theoretical $u$ and $v$ displacements at $x=26 \mathrm{~mm}, y=147 \mathrm{~mm}$ (where $\theta>\cos ^{-1} \frac{S_{L}}{S_{T}}$ ) and $x=141 \mathrm{~mm}, y=51 \mathrm{~mm}$ (where $\theta<\cos ^{-1} \frac{S_{L}}{S_{T}}$ ) generated by a vertical line source which is excited by 5 cycles of sinusoidal function at a frequency of $400 \mathrm{kHz}$ and modulated by a Hanning window. Structural steel is used for the example with the following parameters: $\rho=7850$ $\mathrm{kg} / \mathrm{m}^{3} ; E=2 \mathrm{GPa} ; \sigma=0.33 ; c_{L}=6182 \mathrm{~m} / \mathrm{s} ; c_{T}=3091 \mathrm{~m} / \mathrm{s} ; \mu=75 \mathrm{GPa} ; t=0 \sim 0.07 \mathrm{~ms}$. The analytical solutions will be compared with numerical solution calculated from a finite element (FE) simulation software COMSOL Multiphysics ${ }^{\circledR}$ (COMSOL Inc., Burlington, USA). The blue lines indicate theoretical results and the red dotted lines show FE simulation results. The two kinds of results are consistent with each other. This allows us to calculate the displacements at any point in a half-space excited by linear array. 


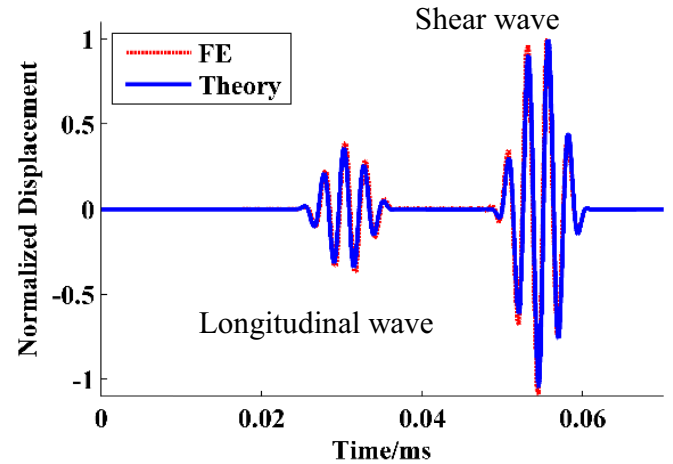

(a) $u$ displacement

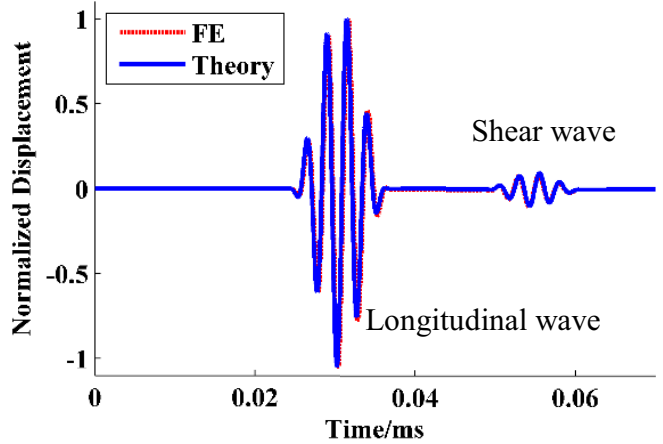

(b) $v$ displacement

Figure 3. Theoretical and simulated $u$ and $v$ displacements at $x=26 \mathrm{~mm}, y=147 \mathrm{~mm}$.

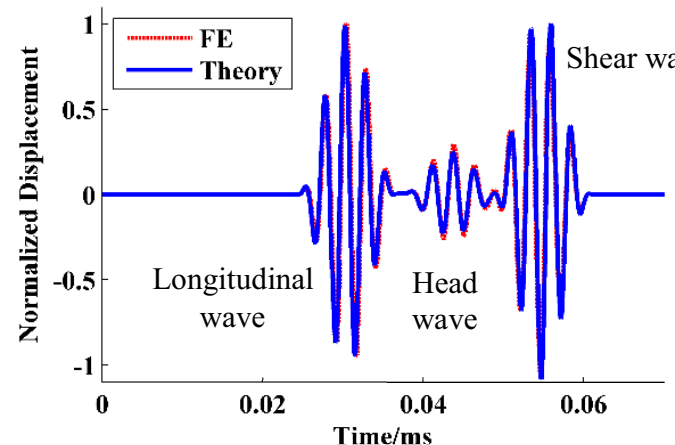

(a) $u$ displacement

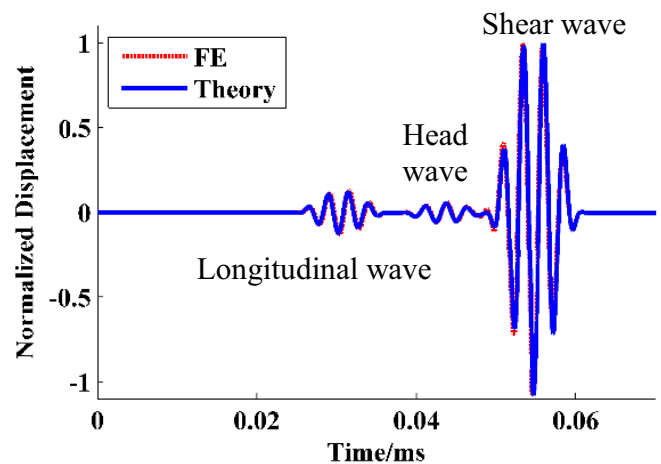

(b) $v$ displacement

Figure 4. Theoretical and simulated $u$ and $v$ displacements at $x=141 \mathrm{~mm}, y=51 \mathrm{~mm}$.

\section{Transient displacements generated by a linear array based on analytical solutions}

The calculation of displacements in a half-space from a linear phased array based on the analytical solutions is discussed below. As shown in Figure 5, each transducer element is modeled by $n$ discrete vertical line sources $(i=1,2, \ldots, n)$, which are uniformly distributed within the transducer element width $(l)$. The $N$ transducer elements are labelled as $j, j=1,2, \ldots, N$. The line sources within one transducer element are excited simultaneously, whereas line sources of different transducer elements will have a given time delay. 


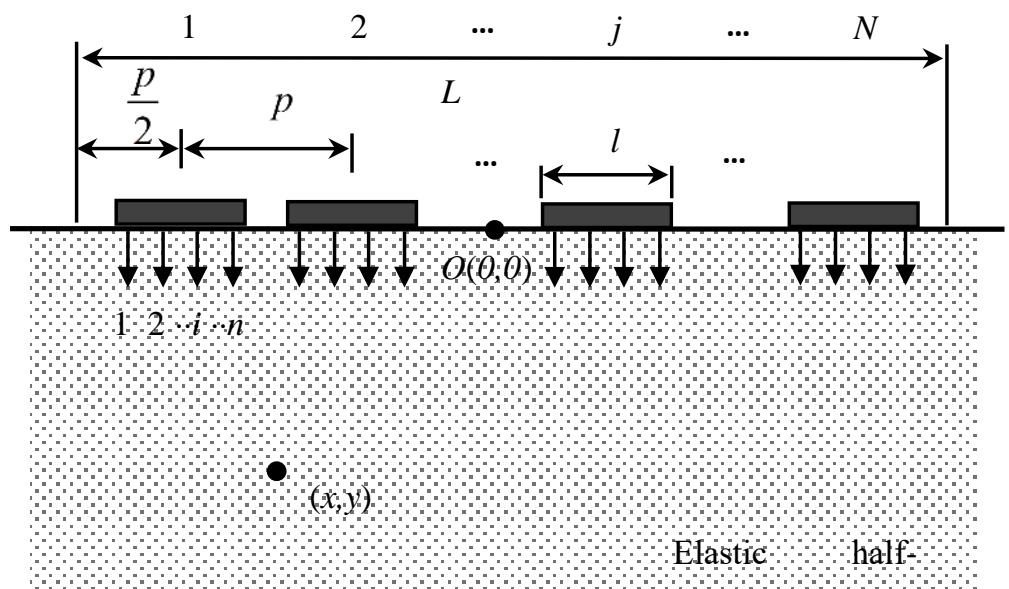

Figure 5. The model of vertical forces loading on an elastic half-space by a linear phased array transducer.

Figure 5 shows the discrete line forces of a linear phased array with an element number $N$, interelement spacing $p$, element width $l$, and array size $L$, and the array is coupled on an elastic half-space with a thin longitudinal wave couplant. As shown in Fig. 5, the coordinate $(x, y)$ is originated from the center of the linear phased array. Let $u_{0}(x, y, t)$ and $v_{0}(x, y, t)$ respectively be the displacement components along $x$ - and $y$-axis of the transient elastic wave radiated by a concentrated line force vertically loaded at the origin point $(0,0)_{(x, y)}$ with a given time domain function of $f(t)$. Based on the superposition principle, the displacements from $j$ th transducer element are

$$
\begin{aligned}
& u_{j}(x, y, t)=\sum_{i=1}^{n} u_{0}\left[x-\left(i-\frac{n+1}{2}\right) \frac{l}{n}-\left(j-\frac{N+1}{2}\right) p, y, t-\Delta t_{j}\right], \\
& v_{j}(x, y, t)=\sum_{i=1}^{n} v_{0}\left[x-\left(i-\frac{n+1}{2}\right) \frac{l}{n}-\left(j-\frac{N+1}{2}\right) p, y, t-\Delta t_{j}\right],
\end{aligned}
$$

where $\Delta t_{j}$ is the time delay assigned to the $j$ 'th transducer element. The displacements from the linear array can be derived,

$$
\begin{aligned}
& U(x, y, t)=\sum_{j=1}^{N} u_{j}(x, y, t), \\
& V(x, y, t)=\sum_{j=1}^{N} v_{j}(x, y, t),
\end{aligned}
$$

where $U(x, y, t)$ and $V(x, y, t)$ are respectively the displacement components along $x$ - and $y$-axis.

Figure 6 shows the theoretical and simulated $u$ and $v$ displacements generated by a linear array at $x=195, y=160$. The excited signal are 6 cycles of sinusoidal function and modulated by a Hanning window.An example with the following parameters are used: $f=200 \mathrm{kHz}, n=20 ; p=20 \mathrm{~mm} ; N=5 ; l=$ $10 \mathrm{~mm} ; t=0 \sim 0.14 \mathrm{~ms}$. The parameters of the steel used here are the same with those of the last example. The blue lines indicate theoretical results and the red dotted lines indicate simulated results. The theoretical results of displacements are consistent with simulated results of displacements. Consequently, this method can provide the accurate transient wave displacements in a half-space from a linear array and replace numerical simulation method, thus saving a lot of computing time. This allows us to calculate the sound field of the linear array, which usually plays a key role in ultrasound inspection. 


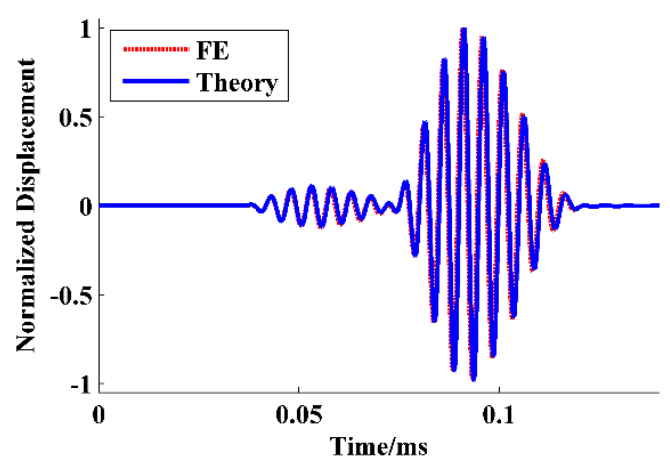

(a) $u$ displacement

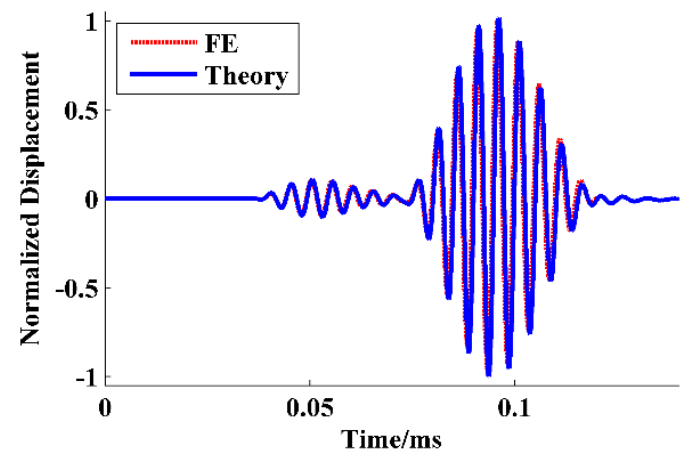

(b) $v$ displacement

Figure 6. Theoretical and simulated $\mathrm{u}$ and $\mathrm{v}$ displacements at $x=195 \mathrm{~mm}, y=160 \mathrm{~mm}$

\section{Conclusion}

This paper proposes a theoretical calculation method for the displacements of transient wave excited by a linear array coupled to an elastic solid with a longitudinal wave couplant. The displacements generated by the linear array mentioned above, are treated as the superposition of the displacements of a number of discrete vertical line forces acting on the surface of a half-space. The displacement amplitude can be easily and accurately calculated at any point in a half-space with minimum assumption and simplification. The theoretical results are consistent with finite element simulation data. Therefore, the method can save a lot of computing time.

This work made improvements for the displacements calculation of transient wave in a half-space from a linear array. It provides precise results for linear array applications. This paper provides a fast and accurate calculation method for the transient displacements in a half-space generated by a linear array. The method can be used to explore the characteristics of the sound field of a linear array transducer.

\section{Acknowledgements}

This work was supported by National Natural Science Foundation of China (grant number 51235001).

\section{References}

1. B.W. Drinkwater, P.D. Wilcox, NDT\&E Int. 39, 525 (2006)

2. M. Spies, J. Nondestruct. Eval. 13, 85 (1994)

3. M. Spies, Ultrasonics 42, 213 (2004)

4. S. C. Wooh, Y. Shi, Wave Motion 29, 245 (1999)

5. S. C. Wooh, Y. Shi, J. Nondestruct. Eval. 18, 39 (1999).

6. S. C. Wooh, Y. Shi, Ultrasonics 36, 737 (1998)

7. L. Azar, Y. Shi, S.C. Wooh, NDT\&E Int. 33, 189 (2000)

8. J. H. Lee, S. W. Choi, IEEE T. Ultrason. Ferr. 47, 644 (2000).

9. J. D. Achenbach, Wave Propagation in Elastic Solids (North-Holland Pub. Co., Amsterdam, 1973) 